\title{
A Biopsy Case of the Adenoameloblastoma
}

\author{
by
}

Yasuyuki $A W A Z A W A^{*}$

The adenoameloblastoma, which is a unique enamel epithelial tumor both in terms of tissue origin and histopathology, presents very different clinical findings from those of an ordinary enamel epithelial tumor. Its incidence is rather infrequent. The present report deals with one case of it which recently came in the experience of the author.

\section{Material and Findings}

The patient, a woman of 23 years, had had no subjective symptoms and, in the course of her dental treatment, a radiolucent image in the size of a quail egg was accidentally found at the apical area of $\overline{432}$. There was no carious lesion or other defect in that area without any loosening of the teeth or percussion pain. Under palpation, the jaw bone of the apical area in question felt to be like a parchment but no change was noted with the mucosa.

An extract from the focus turned out to be a monolocular cystic substance. It presented an appearance similar to that a cyst of the jaw bone and, the outer walls being made up of by fibrous connective tissues, the inside was filled with papilla-like proliferating tissues to about $1 / 4$.

A histological examination revealed a typical adenoameloblastoma. That is, additionally to the structure and epithelial cord more or less resembling an enamel organ, a unique adenoid structure was observed. The focus was nearly solid and gave an appearance of basal cells but, in the central portion, the cells were uniquely arranged in the manner of circles, many of which formed adenoid cavity or pseudoadenoid cavity structures. These adenoid cavities were clear in the majority but a few were found to be filled with hyalinoid substance easily stained by eosin. Inside the tumor substance, many calcium depositions varying in size and shape were present, which could be stained by hematoxylin in different shapes.

*粟沢靖之: Dept. of Pathology, Matsudo College of Dentistry, Nihon University, Matsudo City, Chiba Pref. 
41
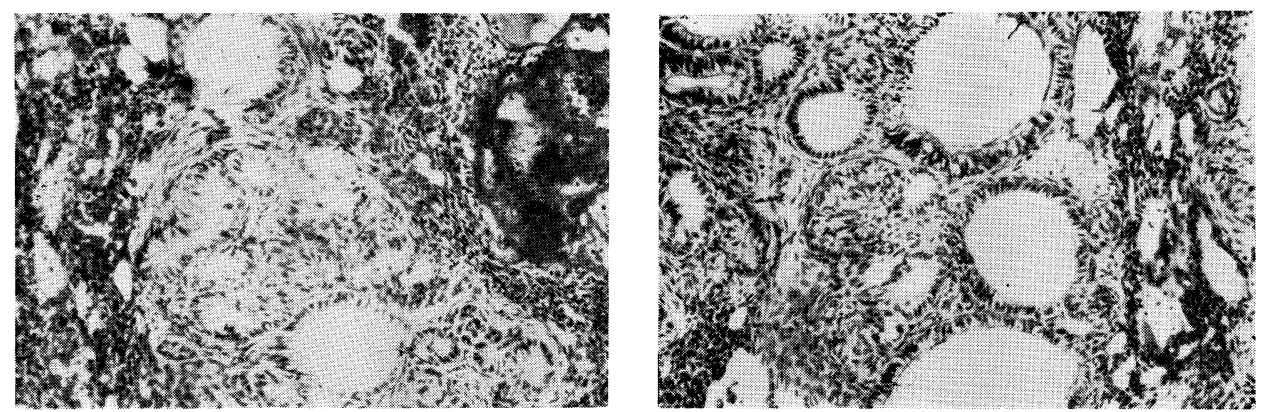

Figs. 1 and 2 Columnar cells arranged in one layer surrond the adenoid cavities in the manner of either circles or tubes.

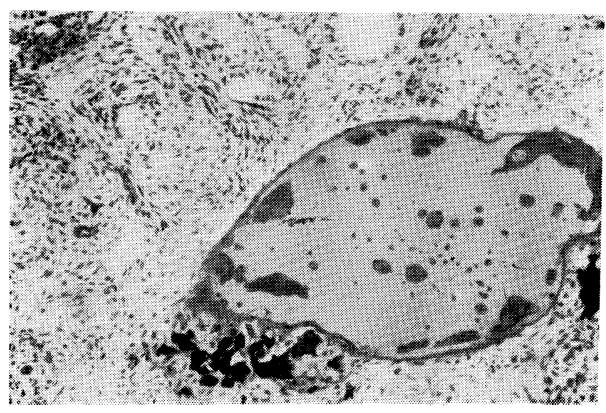

Fig. 3 Calcium depositions are scattered here and there in the tumor tissues.

\section{Discussion}

According to NAKAKuKI et al. (1966), the frequency of this tumor differs by sex and, based on a sample of 37 adeno-ameloblastomata, they give a ratio of $1: 2.1$, the females being more frequent twice as often as the males. The age of 17.9 years is given to be the average year of incidence but the majority of cases occur in their teens, featured by the tendency that, as compared with an ordinary enamel epithelial tumor, this adenoameloblastoma occurs at an earlier age. In terms of jaws, NAKAKUKI et al. give a ratio of 1.5 (upper) and 1.0 (lower) and being mostly confined to the anteriors and premolars, it is very rare in the molars with a ratio of $1: 18$. This last feature deserves our attention in particular, for an ordinary enamel epithelial tumor favors the molar region instead.

\section{Reference}

Nakakuki, T.: A case of adenoameloblastoma, Shikagakuho, Vol. 66, p. 82, (1966), (Japanese text). 